\title{
Pancytopenia due to graft versus host disease post liver transplantation: A case report
}

\author{
Uzoka C', Tzvetanov IG ${ }^{2}$, Patel P1, Benedetti E ${ }^{2}$ and Rondelli D ${ }^{1}$ \\ ${ }^{1}$ Department of Medicine, Hematology/Oncology Division, University of Illinois, Chicago, IL USA \\ ${ }^{2}$ Department of Surgery, Transplant Division, University of Illinois, Chicago, IL USA
}

\begin{abstract}
Background: Cytopenias can have a huge impact on patients' outcome in the immediate post solid organ transplant period. Less frequently it is caused by acute GVHD, and the treatment is usually different from the more common causes of cytopenias like infections and medications.

Materials and Method: We report a case of profound pancytopenia due to GVHD, in a 26-year-old female recipient of an orthotopic liver transplantation, following acute liver failure. Bone marrow biopsy features of aplastic anemia, a skin biopsy showing apoptotic keratinocytes with focal lymphocytic infiltrates and a mixed chimerism pattern of $40 \%$ myeloid donor cells, were consistent with acute GVHD in this context. The aplastic bone marrow features were likely prompted by the donor allo-reactivity against the recipient bone marrow cells.

Results: She was treated with high dose steroids and transfusion support. An allogeneic hematopoietic stem cell transplant was considered, in the event, where the aplastic anemia was refractory to steroids and an appropriate stem cell donor was identified. She responded to immune suppression with platelet and neutrophil count improvement, but unfortunately died 2 months later from intracranial hemorrhage.

Conclusion: GVHD, in this setting, though infrequently reported, has a very high mortality. Various therapeutic modalities are inconsistent; hence a unified treatment and monitoring strategy is advocated.
\end{abstract}

\section{Introduction}

Decrease of one or multiple blood cell lineages (cytopenia) following solid organ transplant (SOT) can severely affect patients' outcome by increasing the risk of infection and hemorrhage, development of anti-red blood cell antibodies and overall mortality. Cytopenia is more commonly observed within 2-6 weeks post SOT, and potential implicating factors include: infections, autoimmune responses, or drug-associated myelotoxicity. In particular, immunosuppressive agents commonly utilized in transplantation, such as azathioprine, cyclosporine, tacrolimus, sirolimus, mycophenolate, and some antimicrobials (sulfamethoxazole, dapsone, valganciclovir) have all been associated with cases of cytopenias [1-4].

Another rare cause of post-SOT cytopenia can be graft-versus-host disease (GVHD). This occurs when transplanted grafts (particularly liver and small bowel) contain large number of donor T lymphocytes that recognize host alloantigens, differentiate into effector cells and attack recipient tissues [5]. Similarly, in the 'Passenger Lymphocyte Syndrome', B cells present in the donor graft expand in the recipient and produce anti-red blood cell (anti-RBC) antibodies that cause severe hemolytic anemia $[6,7]$.

The diagnosis of GVHD post SOT can be associated with any of its classic symptoms: skin rash, diarrhea, elevated bilirubin and/or liver enzymes, as well as bone marrow aplasia. The latter is diagnosed with a bone marrow biopsy, which demonstrates the presence of donorderived cells by molecular analysis (chimerism). Viral infections from Epstein-Barr virus (EBV), cytomegalovirus (CMV), or parvovirus B19 which can cause aplasia can also be tested. Although the treatment of post SOT GVHD has not been standardized, the empiric use of corticosteroids and intensification of immunosuppression are the main treatment modalities, which is similar to the treatment of GVHD post stem cell transplant.

Here we report a case of a patient who developed severe cytopenia following an orthotopic liver transplant (OLT), later diagnosed to be related to GVHD, and we review treatment options reported in the literature.

\section{Case Report}

A previously healthy 26-year-old woman presented at the hospital with acute liver failure, progressively worsening, with a Model for End-stage Liver Disease (MELD) score of 31. She received an OLT from a 52-year-old ABO compatible cadaveric donor. Induction immunosuppression included basiliximab and a steroid taper, while immune suppression was maintained with tacrolimus and Mycophenolate mofetil per organ transplant protocol [8]. Her immediate post-operative course was characterized by prolonged respiratory failure, abdominal compartment syndrome, multiple organ failure from postoperative days (POD) 0 - 14 . In the $3^{\text {rd }}$ week after the SOT, she improved clinically, with recovery of the liver allograft function.

${ }^{\star}$ Correspondence to: Chukwuemeka Uzoka, Division of Hematology/Oncology, Department of Medicine, University of Illinois, Chicago, 840 S Wood St, 820-E CSB, MC 713, Chicago, IL 60612, USA, Tel: 312-996-1581, E-mail: Uzokac18@ ecu.edu

Received: January 21, 2019; Accepted: January 28, 2019; Published: January 31, 2019 
On POD 38, she started developing cytopenia, with absolute neutrophil count of $0.6 \times 10^{9} / \mathrm{L}$, and requiring multiple transfusions to maintain the levels of hemoglobin above $8 \mathrm{~g} / \mathrm{dl}$ and platelets above $50 \times 10^{9} / \mathrm{L}$ (Figure 1). A bone marrow biopsy revealed a markedly hypocellular marrow ( $<5 \%$ cellularity) with no features of dysplasia (Figure 2 ). Molecular studies were negative for CMV, parvovirus, varicella zoster (VZV), herpes viruses, (HSV I/II), and adenovirus, while showed only a few copies of EBV mRNA. Medications potentially myelosuppressive, such as dapsone, valganciclovir and mycophenolate were held, and filgrastim to stimulate myeloid recovery was administered, but no improvement in the blood counts was observed. On POD 42, the patient developed a skin rash in the chest and both upper extremities. A skin biopsy at the site of the rash revealed scattered isolated apoptotic keratinocytes with patchy minimal pigment incontinence and focal lymphocytic satellitosis, consistent with skin acute GVHD grade I-II. Since we could not have pre-transplant cells from the cadaveric donor, chimerism studies were performed via single tandem repeat (STR) analysis by comparing cells from the patient's buccal mucosa with those obtained from the peripheral blood. The results from circulating peripheral blood cells showed a mixed chimerism pattern, with $12 \%$ donor total mononuclear cells. The analysis in myeloid and T lymphoid subsets revealed $40 \%$ donor $\mathrm{CD}^{+} 3^{+}$myeloid cells, and $7 \%$ donor $\mathrm{CD}^{+}$ $\mathrm{T}$ cells. These findings of donor-derived myeloid and $\mathrm{T}$ cells in the context of a hypocellular bone marrow suggested that the marrow aplasia could be possibly caused by donor allo-reactivity against the recipient bone marrow.
Treatment options that were considered at that time included increase of immune suppression and, if not successful, eventually plan for allogeneic bone marrow transplant, should a donor be identified. On POD 45, the patient was started on prednisone at the standard dose for acute GVHD of $2 \mathrm{mg} / \mathrm{kg} /$ day. By POD 57, the skin rash improved and the absolute neutrophil count reached normal levels (Figure 1). However, the platelet number never recovered beyond 72,000 by POD 60 (Figure 1), and she continued to require RBC transfusions. On POD 99, the patient was discharged from the hospital and continues to receive transfusions in the outpatient. However, approximately 2 months later she died of intracranial bleeding.

\section{Discussion}

Our report describes the case of a young patient who developed acute GVHD grade 1 and severe cytopenia, 5 weeks after OLT. Post SOT GVHD has been observed more frequently in small bowel and liver transplants, closely followed by lung and kidney transplants [9]. In OLT, it was previously shown that the transfer of approximately $10^{9}-10^{10}$ donor $\mathrm{T}$ lymphocytes in the graft, remaining in the portal tract after flushing with preservation solution, could be activated by alloantigens and cause GVHD [10].

Moreover, T cells also release pro-inflammatory cytokines such as IL-2, IL-18 and IFN- $\gamma$, that have been characterized as early diagnostic biomarkers of GVHD in liver graft recipients by Meng, et al. [11]. Although the incidence of SOT GVHD reported by the United Network
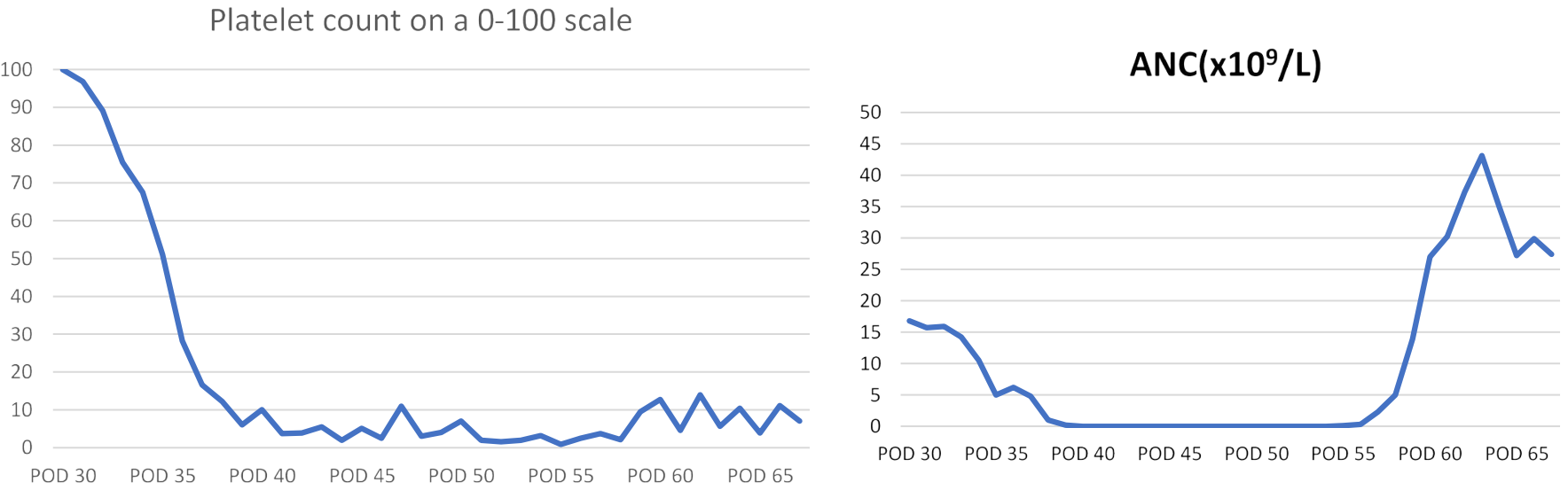

$\mathrm{Hb}(\mathrm{g} / \mathrm{dl})$

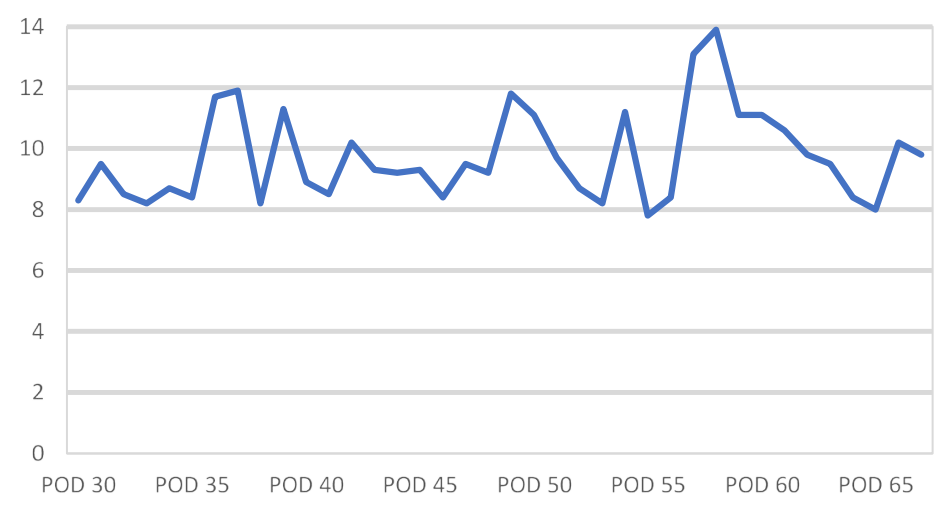

Figure 1. Graphical representation of decline of all 3 cell lines 


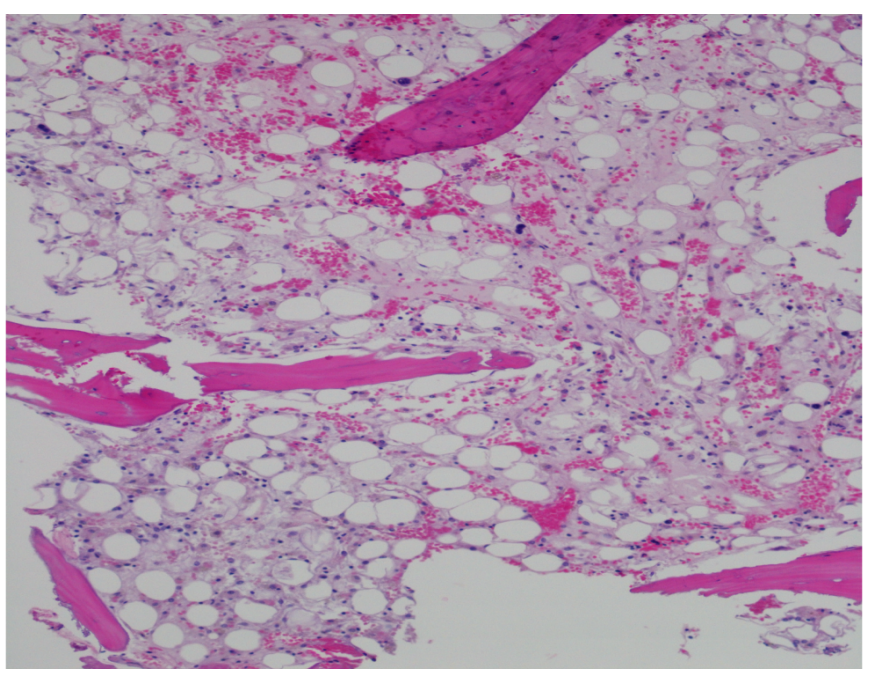

Figure 2A. H\&E stain at 100x magnification showing an aplastic marrow devoid of hematologic precursors

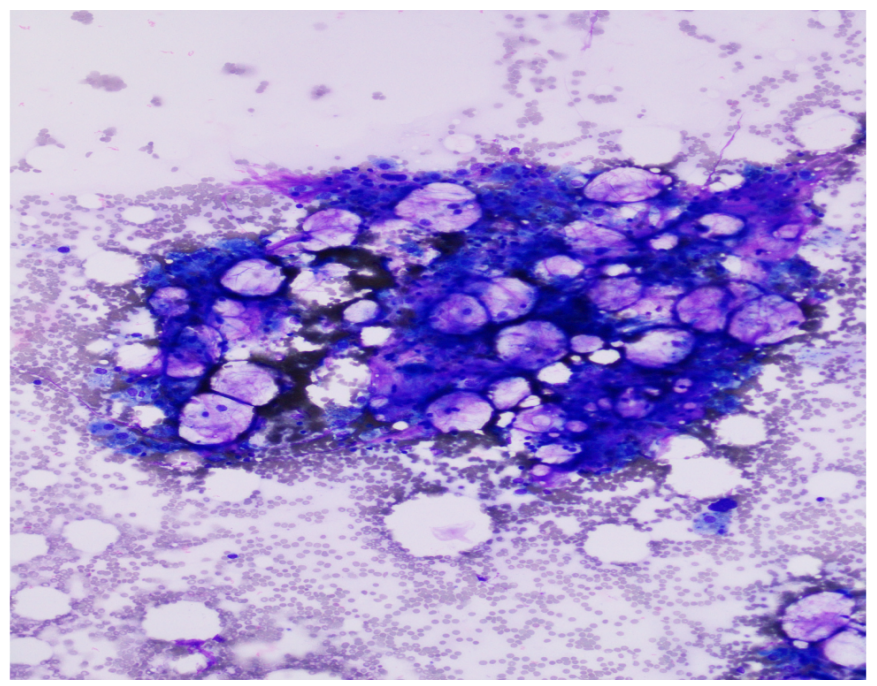

Figure 2B. Wright Giemsa stain at 200x magnification showing the same aplastic marrow devoid of hematologic precursors

for Organ Sharing (UNOS) is only $0.1 \%$, other reports indicate a higher incidence estimated at $1-2 \%[9,12]$. This difference could be due to underreporting, or to symptoms wrongly attributed to other causes than GVHD. Five main risk factors have been associated with SOT GVHD: a) recipient age greater than 50 years; b) donor - recipient age difference greater than 20 years; c) younger donor age; d) HLA class I mismatch; e) glucose intolerance [12,13]. In our case, a risk factor was the donor- recipient age difference of 26 years.

Features of GVHD such as skin rash, cytopenia and diarrhea occur usually within 38 days' post LT, and cytopenia has been described to confer a worse outcome [14]. In our case, the diagnosis of GVHD was supported by clinical findings and also by the donor cell chimerism found in the peripheral blood. This was consistent with previous reports showing that the detection of donor chimerism $>1 \%$ in the first few weeks post LT is more often associated with GVHD $[15,16]$.

Treatment outcomes of GVHD post liver transplant tend to be poor, with $>85 \%$ mortality rate $[10,15]$. This is in part due to the high degree of HLA mismatch between patient and donor, as well as by complications of cytopenias in the post-transplant setting, such as sepsis, multi-organ failure, or bleeding [14].

In our patient, increased immunosuppression with high dose steroids, improved the neutrophil count, but the platelets didn't fully recover. The lack of clinical trials for SOT GVHD treatment limits an effective therapeutic strategy. Interestingly, either decreased or increased levels of immunosuppression have been proposed, with the assumption that either blocking the donor GVH response (increasing immunosuppression) or enhancing the recipient host versus graft response causing graft rejection (withdrawing immunosuppression), could be beneficial to stop the GVHD [17-19]. Treatment with steroids and anti-thymocyte globulin (ATG) based regimens, with or without calcineurin inhibitors, were associated with mortality rates over $70 \%$ in patients with GVHD post LT [14]. Regimens with the CD2 inhibitor alefacept, steroids and ATG as a means of T cell depletion, showed less mortality but these studies were limited by a small sample size [20-22]. Allogeneic hematopoietic stem cell transplantation (HSCT) was also previously performed in a few pediatric case reports with prolonged post SOT cytopenia [23,24]. However the risks of infection and chemotherapy related toxicity after HSCT should be carefully assessed in the post SOT setting.

In conclusion, GVHD after OLT is associated with poor outcomes and a high mortality rate. Analysis of donor macro-chimerism and cytokines levels may be helpful for early diagnosis and monitoring of response to treatment. Though case reports and small series have utilized multiple immunosuppressive agents, multicenter prospective studies for treatment of SOT GVHD are warranted.

\section{Acknowledgments}

The authors have no conflict of interest to disclose. C. Uzoka and D. Rondelli wrote the manuscript and all the authors contributed to the revision of the manuscript.

\section{References}

1. Danesi R, Del Tacca M (2004) Hematologic toxicity of immunosuppressive treatment. Transplant Proc 36: 703-704.

2. Brum S, Nolasco F, Sousa J (2008) Leukopenia in kidney transplant patients with the association of valganciclovir and mycophenolate mofetil. Transplant Proc 40: 752-754.

3. Hong JC, Kahan BD (2000) Sirolimus-induced thrombocytopenia and leukopenia in renal transplant recipients: risk factors, incidence, progression, and management. Transplantation 69: 2085-2090. [Crossref]

4. Danziger-Isakov L, Mark Baillie G (2009) Hematologic complications of anti-CMV therapy in solid organ transplant recipients. Clin Transplant 23: 295-304.

5. Pollack MS, Speeg KV, Callander NS (2005) Severe, late-onset graft-versus-host disease in a liver transplant recipient documented by chimerism analysis. Hum Immunol 66: 28-31.

6. Chua CC, Lim H, Testro A, Hong FS (2018) Passenger lymphocyte syndrome due to anti-B and anti-Jka following combined intestinal and renal transplantation. ISBT.

7. Panaro F, Rondelli D, Benedetti E (2004) Severe hemolytic anemia due to passenger lymphocytes after living-related bowel transplant. Clin Transplant 18: 332-335.

8. Ramirez CB (2006) Basiliximab Induction in Adult Liver Transplant Recipients with 93\% Rejection-Free Patient and Graft Survival at 24 Months. Transplant Proc 38: 3633-3635.

9. Smith DM Agura E, Netto G (2003) Liver transplant-associated graft-versus-host disease. Transplantation 75: 118-126.

10. Kohler S, Pascher A, Junge G (2008) Graft versus host disease after liver transplantation-a single center experience and review of literature. Transpl Int 21: 441-451.

11. Meng XQ, Chen XH, Sahebally Z, Xu YN, Yin SY, et al. (2017) Cytokines are early diagnostic biomarkers of graft-versus-host disease in liver recipients. Hepatobiliary Pancreat Dis Int 16: 45-51. [Crossref] 
12. Chan EY, Larson AM Gernsheimer TB (2007) Recipient and donor factors influence the incidence of graft-vs.-host disease in liver transplant patients. Liver transpl 13: 516-522.

13. Elfeki MGP, Pungpapong S, Nguyen J, Harnois D, Graft-Vs-Host, et al. (2015) Disease after Orthotopic Liver Transplantation: Multivariate Analysis of risk factors. $\mathrm{Am} J$ transplant $\mathrm{p} .15$.

14. Murali (2016) Graft versus Host Disease After Liver Transplantation in Adults: A Case series, Review of Literature, and an Approach to Management. Transplantation 100:2661-2670.

15. Taylor AL, Gibbs P, Sudhindran S (2004) Monitoring systemic donor lymphocyte macrochimerism to aid the diagnosis of graft-versus-host disease after liver transplantation. Transplantation 77: 441-446.

16. Domiati-Saad R, Klintmalm GB, Netto G (2005) Acute graft versus host disease after liver transplantation: patterns of lymphocyte chimerism. Am J Transplant 5: 29682973.

17. Lehner F, Becker T, Sybrecht L, Luck R, Schwinzer R, et al. (2002) Successful outcome of acute graft-versus-host disease in a liver allograft recipient by withdrawal of immunosuppression. Transplantation 73: 307-310.
18. Wang B, Lu Y, Yu L, Liu C, Wu Z, et al. (2007) Diagnosis and treatment for graftversus-host disease after liver transplantation: two case reports. Transplant Proc 39: 1696-1698. [Crossref]

19. Chinnakotla S, Smith DM, Domiati-Saad R (2007) Acute graft-versus-host disease after liver transplantation: role of withdrawal of immunosuppression in therapeutic management. Liver Transpl 13: 157-161.

20. Rogulj IM, Deeg J, Lee SJ (2012) Acute graft versus host disease after orthotopic liver transplantation. J Hematol Oncol 5: 50.

21. Stotler CJ, Eghtesad B, Hsi E (2009) Rapid resolution of GVHD after orthotopic liver transplantation in a patient treated with alefacept. Blood 113: 5365-5366.

22. Eghtesad B, Askar M, Bollinger J (2012) Graft-versus-host disease (GVHD) following liver transplantation (LT): Comparison of two eras. Am J Transplant 12: 126.

23. Stachel D (2002) Double bone marrow transplantation for severe aplastic anemia after orthotopic liver transplantation: implications for clinical management and immune tolerance. Transpl Int 15: 39-44

24. JL Perkins, JP Meglia (2001) Successful bone marrow transplantation for severe aplastic anemia following orthotopic liver transplantation: long-term follow-up and outcome. Bone Marrow Transplant 28: 523-526.

Copyright: (O2019 Uzoka C. This is an open-access article distributed under the terms of the Creative Commons Attribution License, which permits unrestricted use, distribution, and reproduction in any medium, provided the original author and source are credited. 